\title{
Ovarian metastasis after radical nephrectomy for primary renal clear cell carcinoma: a diagnostic challenge
}

\author{
Mathieu Luyckx • Jacques Donnez • Monique Delos • \\ Joseph Kerger • Olivier Donnez
}

Received: 2 April 2010 /Accepted: 18 May 2010 /Published online: 12 June 2010

(C) Springer-Verlag 2010

Keywords Ovary · Metastasis · Renal clear cell carcinoma

\section{Introduction}

Renal clear cell carcinoma (RCC) can lead to metastases in unusual sites, but rarely in the ovary [1]. Differentiation between primary ovarian tumors and ovarian metastases from RCC has different prognostic and therapeutic implications. As ovarian metastasis from RCC resembles a primary ovarian tumor, diagnosis often presents a histopathological challenge [1].

Immunohistochemistry has proved very useful in distinguishing primary ovarian lesions from RCC metastases in the ovary, generally using CK7, CK20, vimentin, ER and CA125 [2].

There are 15 documented cases in the English literature of ovarian metastases developing from RCC confirmed by immunohistochemistry, often discovered during follow-up of the primary renal carcinoma. The search engine used for the literature review was PUBMED with "renal clear cell carcinoma", "ovary", and "ovarian metastasis" as key words.

\footnotetext{
M. Luyckx $\cdot$ M. Delos $\cdot$ J. Kerger $\cdot$ O. Donnez

Department of Gynecology, Cliniques Universitaires

de Mont-Godinne, Université Catholique de Louvain,

7430 Yvoir, Belgium

J. Donnez $(\bowtie)$

Department of Gynecology, Cliniques Universitaires Saint-Luc,

Université Catholique de Louvain,

Avenue Hippocrate, 10,

1200 Brussels, Belgium

e-mail: Jacques.donnez@uclouvain.be
}

Here we present the 16th case of RCC metastasis in the ovary, first appearing as highly hemorrhagic tumors.

\section{Case presentation}

A 75-year-old Caucasian woman was referred to our department with an adnexal mass in the pelvis, which was increasing in size. She had never been pregnant and was menopausal at 52 years of age. She had never had any gynecologic examinations. Her medical history was characterized by hypothyroidism, hypertension, and alcohol addiction. At the age of 70 , she had undergone a radical nephrectomy for a heterogeneous mass situated on the lower pole of the left kidney. The histopathological findings revealed low-grade RCC with perforation of the kidney capsule. None of the five lymph nodes showed any metastasis and the suprarenal gland was normal. No adjuvant treatment was given after surgery. After 1 year of follow-up, CT scan of the abdomen revealed multiple hepatic lesions disseminated in the right lobe, associated with multiple pulmonary lesions. Both the hepatic and pulmonary lesions were suspected to be metastases from the RCC, but this was never confirmed by biopsy. A right lateral uterine lesion of $4 \times 3 \mathrm{~cm}$ corresponding to a liquid cyst in the right anexa was also described and considered to be benign. The patient first received interleukin-2 with a very good pulmonary response and stabilization of the hepatic lesions but, because of significant side effects (acute kidney failure), treatment was discontinued. Interferon and vinblastine were then administered. Six months after initiation of this treatment, CT scan revealed progression of the hepatic lesions. Interleukin-2 therapy was reinstated at lower doses for 1 year and was well tolerated. The right adnexal lesion was stable at this time. The hepatic 
lesions also stabilized, while the pulmonary lesions were undetectable on $\mathrm{CT}$ scan. The woman underwent regular $\mathrm{CT}$ scan controls and the adnexal lesion remained stable in size. However, at the age of 75 years, the CT scan result revealed that the right adnexal mass had grown to $10 \mathrm{~cm}$ in size within 5 months. This mass was heterogeneous with a highly vascularized cyst wall and septa (Fig. 1a, b). She was then referred to our gynecology department.

Upon vaginal examination, a $10-\mathrm{cm}$ retro-uterine solid mass was found fixed to the Douglas pouch. Ultrasound confirmed the presence of a heterogeneous right ovarian mass, with extensive vascularization. The CA125 level was $11.3 \mathrm{IU} / \mathrm{ml}(\mathrm{nl}<35 \mathrm{IU} / \mathrm{ml})$.

Diagnostic laparoscopy was then proposed. The internal status revealed a normal liver, unremarkable left adnexa, and a $10-\mathrm{cm}$ lesion on the right ovary, with dense adhesions to the rectum and uterus. No ascites was found in the abdomen. Macroscopically, the tumor had a smooth surface without any vegetation. Radical hysterectomy with bilateral
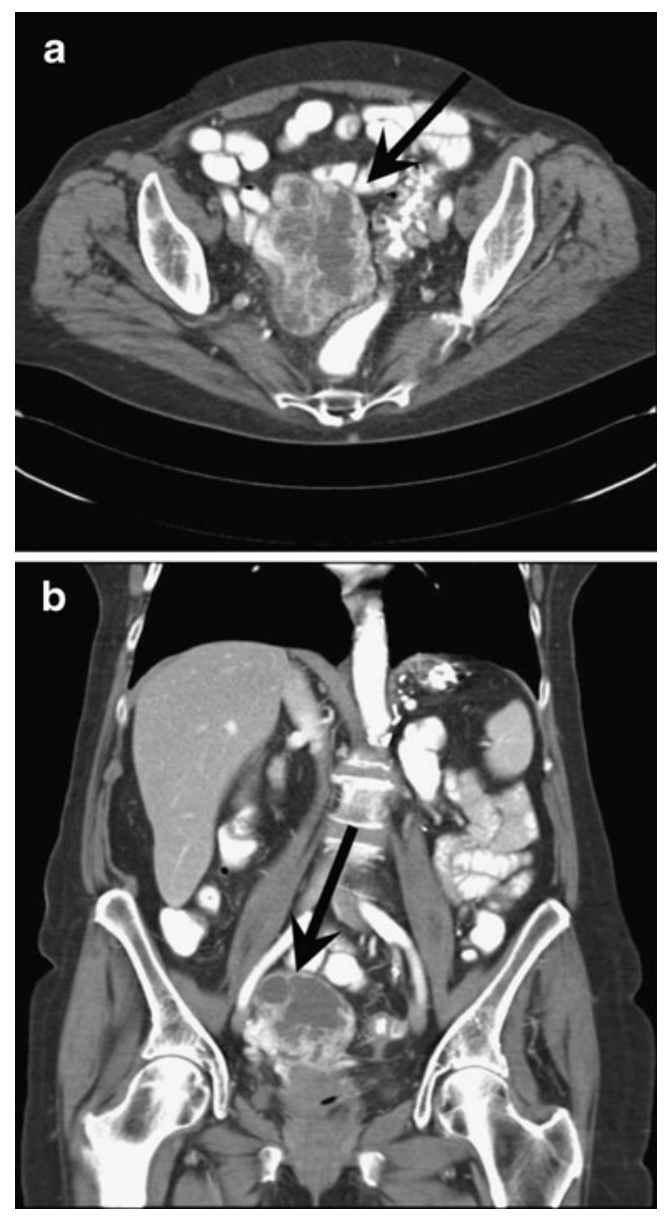

Fig. 1 a CT scan revealed a 10-cm adnexal lesion (black arrow). The mass was heterogeneous and highly vascularized, clearly distinct from the bowel and pelvic side wall. There was no ascites or peritoneal spread. b Coronal view of the CT scan shows the right adnexal mass (black arrow)

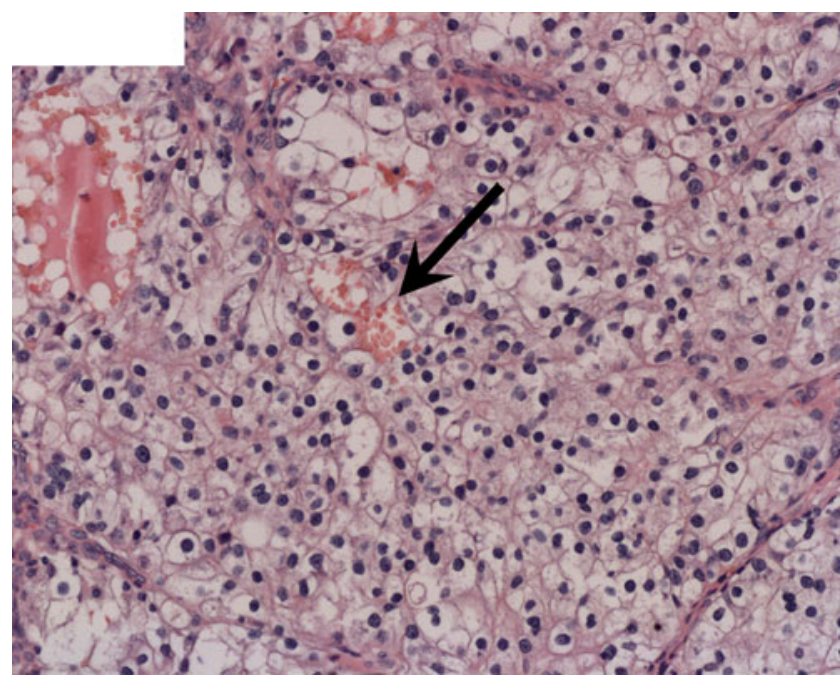

Fig. 2 Hematoxylin-eosin staining revealed cells arranged in nests and cords with clear and abundant cytoplasm. The black arrow shows the dense capillary network of the lesion

salpingo-oophorectomy by laparotomy was therefore performed. Frozen section revealed metastasis of the RCC.

Macroscopically, a solid yellow tumor and a large zone of necrosis were visualized. Microscopically, the lesion showed large areas of necrosis and hemorrhage. It was also found to be composed of neoplastic cells arranged in nests or cords and characterized by clear and abundant cytoplasm with atypical nuclei (Fig. 2). An extensive capillary network was observed to be involved in the tumoral proliferation. The tubes, left adnexa, and uterus were all unremarkable.

This appearance suggested that the mass was ovarian metastasis from the RCC from the previously removed left kidney. Immunohistochemical analysis showed the tumor to be positive for CD10 (Fig. 3) and CK8.8, while CK7, CA125, and ER were negative. These immunohistological findings confirmed the diagnosis of ovarian metastasis from the RCC.

No adjuvant treatment was given. CT scans performed every 6 months have been stable so far and, 1.5 years after the ovarian surgery (and 6 years after the removal of the primary renal lesion), the patient is still alive without any local recurrence or evolution of the hepatic or pulmonary lesions.

\section{Discussion}

Metastasis from RCC tumors occurs rarely in the ovaries. The most commonly encountered secondary neoplasms in the ovaries are colonic adenocarcinoma, breast carcinoma, lymphoma, carcinoid adenocarcinoma, and gastric adenocarcinoma, which macroscopically and microscopically resemble primary ovarian cancer [3].

This infrequent occurrence may be explained by some physiological factors; RCC is more frequently found in 

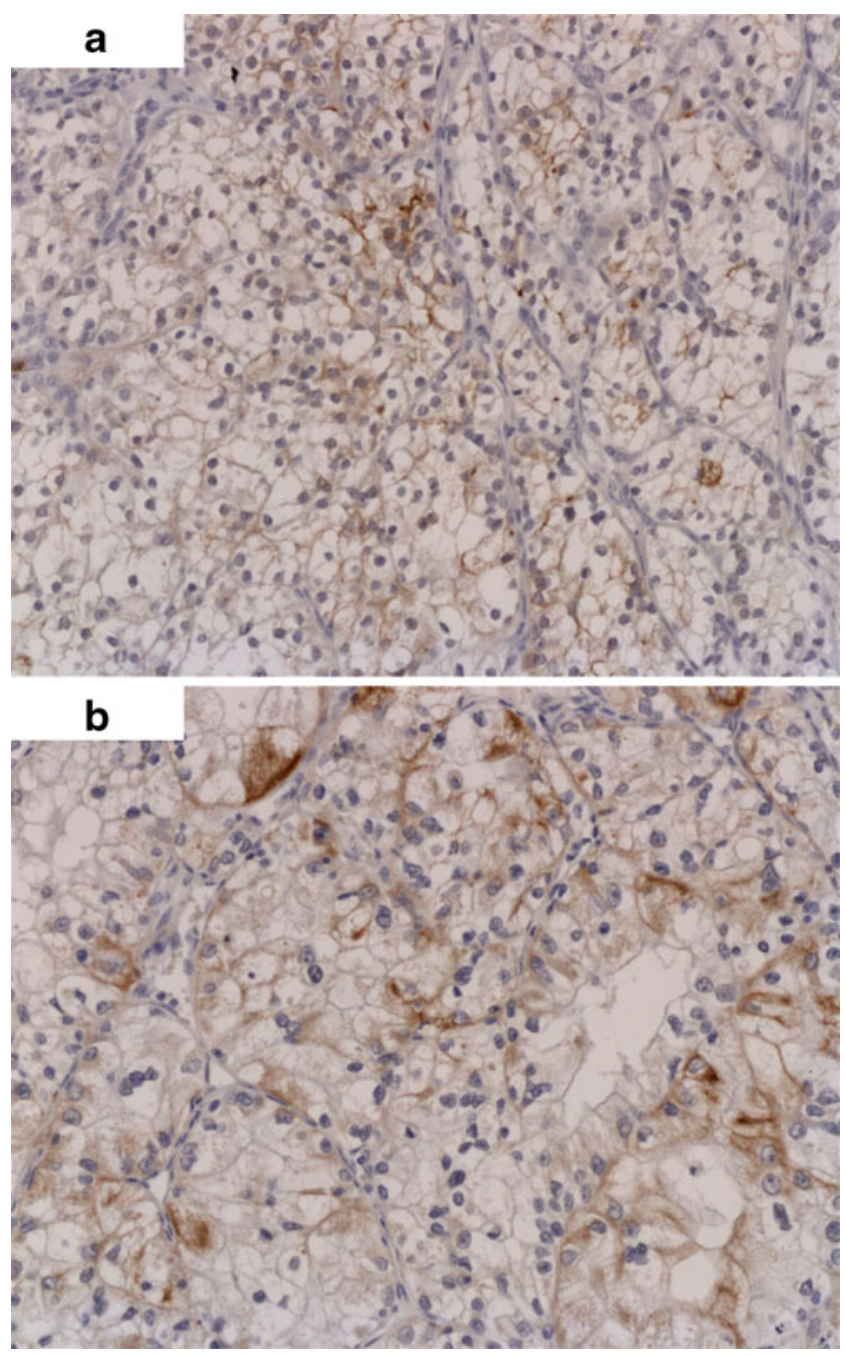

Fig. 3 Immunohistochemical analysis. a Immunoassay for CD10 was positive. b Immunoassay for Cytocam 5.2 was positive

males and usually in older patients [4], while vascularization of the ovaries is decreased in older women because of atrophy due to menopause. However, Gavallos et al. suggested that a connection between the kidneys and the ovaries could allow interchange of malignancies between them [5]. They suggested the possibility of a renal-ovarian axis. Indeed, some autopsy studies demonstrated that $0.5 \%$ of women with RCC showed ovarian metastasis, often undiagnosed, and $4.2 \%$ of secondary ovarian lesions were of renal origin [6]. Metastasis from the ovary to the kidney is much less frequent with only two cases described [5].

There are currently 15 cases of ovarian metastasis from RCC documented in the English literature, with available immunohistochemistry $[7-9,11,14,20-25]$, and we now report the 16th case. These 16 cases have been analyzed and are summarized in Table 1.

With an age range of 17 to 75 years, the present case is actually the oldest patient described with this type of ovarian metastasis. In $31 \%$ of cases $(n=5 / 16)$, the ovarian lesion was detected first and the time needed to detect the primary renal lesion was very varied. Generally, when the ovarian lesion was detected first, the renal lesion was located soon after. In $12.5 \%$ of cases $(n=2 / 16)$, there was synchronous detection by imaging or during surgery. In another two cases, the time was respectively 7 months and a few weeks after ovarian surgery. In one case, the primary renal lesion was detected 8 years after ovarian surgery [7-9].

When the ovarian lesion was discovered after the renal lesion, the time interval to the appearance of the ovarian metastasis ranged from 3 months to 3 years.

Two other cases showed a much longer interval, from 5 to 14 years after primary renal lesion detection. In the case we describe here, the ovarian metastasis appeared 4 years after the development of hepatic and pulmonary lesions. These lesions have now been stable for 4 years.

The survival rate after ovarian metastasis is very varied, ranging from a few months to 8 years, but is often not mentioned.

Gavallos et al. discussed possible pathways of metastasis from the kidney to the ovaries [5]. Direct peritoneal seeding is not typical of RCC and will not result in unique and well delimited metastasis. There is no evidence of lymphatic dissemination either. The most common way for RCC to spread to other organs is by the hematogenous route. On the other hand, Young et al. and Bruegge et al. discussed the unique anatomy of the left ovarian veins and their relationship with the left renal veins $[4,9]$. The hypothesis was that, in case of incompetent gonadal veins allowing retrograde flow, renal tumors could spread to the left ovary.

On the basis of this hypothesis, the laterality of both types of lesions was analyzed: of the 16 described cases, $69 \%$ showed a left renal lesion $(n=11 / 16)$. Ovarian metastasis from renal lesions appears to be more frequent when the primary lesion is from the left kidney.

Among those 11 cases, $45 \%(n=5 / 11)$ had a left ovarian lesion, 73\% $(n=8 / 11)$ bilateral metastases, and 27\% $(n=3 / 11$, including our case) a right ovarian lesion.

Among the other five cases presenting with right renal lesions, $60 \%(n=3 / 5)$ had a left ovarian lesion, one was unknown, and one showed right ovarian metastasis $(20 \%)$.

We identified a trend in the metastatic process: left renal lesions metastasized most commonly to the left ovary, and even with right renal lesions, ovarian metastasis was more frequently observed in the left ovary. These findings support the theory of hematogenous spread from the renal tumor to the incompetent left gonadal veins to the ovary, even if it cannot be the only mechanism of metastasis.

Another particularity of RCC metastasis to the ovary is the relative rarity of bilateral ovarian involvement. Although Petru et al. found $60-70 \%$ of secondary ovarian 
Table 1 Review of all cases of ovarian metastasis from RCC in the English literature

\begin{tabular}{|c|c|c|c|c|c|c|c|}
\hline \multirow{2}{*}{$\begin{array}{l}\text { Publication } \\
\text { year }\end{array}$} & \multirow[t]{2}{*}{ Author } & \multirow{2}{*}{$\begin{array}{l}\text { Age } \\
\text { (years) }\end{array}$} & \multirow{2}{*}{$\begin{array}{l}\text { First } \\
\text { detected }\end{array}$} & \multicolumn{2}{|l|}{ Laterality } & \multirow{2}{*}{$\begin{array}{l}\text { Time to diagnosis of } \\
\text { other lesions }\end{array}$} & \multirow[t]{2}{*}{ Outcome after last surgery } \\
\hline & & & & Ovarian & Renal & & \\
\hline 1981 & Stefani et al. [20] & 39 & Kidney & Left & Right & 3 months & Alive $>2$ years \\
\hline 1983 & Buller et al. $[21]^{0}$ & 52 & Ovary & Left & Left & Synchronous & Unknown \\
\hline 1992 & Young et al. [9] & 48 & Ovary & Left & Right & 8 years & Unknown \\
\hline 1992 & Young et al. [9] & 62 & Kidney & Right & Left & 1 year & Unknown \\
\hline 1992 & Young et al. [9] & 48 & Ovary & Left & Left & Synchronous & Unknown \\
\hline 1993 & Spencer et al. [7] & 40 & Ovary & Bilateral & Left & 7 months & Alive $>4$ years with disease \\
\hline 1994 & Adachi et.al [22] & 46 & Kidney & Bilateral & Left & 3 years & Alive $>3$ years \\
\hline 1996 & Fields et al. [23] & 40 & Kidney & Left & Left & 3 years & Alive 4 years with disease spread \\
\hline 1998 & Vara et al. [24] & 57 & Kidney & Left & Left & 14 years & Died 18 months \\
\hline 2003 & Hammock et al [8] & 48 & Ovary & Right & Left & A few weeks & Unknown \\
\hline 2003 & Insabato et al. [11] & 50 & Kidney & Right & Right & 1 year & Lost to follow-up \\
\hline 2003 & Insabato et al. [11] & 49 & Kidney & - & Right & 14 months & Died 20 months \\
\hline 2003 & Insabato et al. [11] & 17 & Kidney & Left & Left & 2 years & Alive $>24$ months \\
\hline 2004 & Valappil et al. [14] & 61 & Kidney & Bilateral & Left & 7 years & Alive 2 years \\
\hline 2007 & Stolnicu et al. [25] & 73 & Kidney & Left & Right & 3 years & Unknown \\
\hline 2009 & $\begin{array}{l}\text { Luyckx et al. } \\
\text { [present study] }\end{array}$ & 75 & Kidney & Right & Left & 5 years & Alive $>6$ years with stable meta. \\
\hline
\end{tabular}

tumors to be bilateral [10], this was clearly not the case in our review $(19 \%, n=3 / 16)$.

Hammock et al. and Insabato et al. detailed and reviewed the differential diagnoses for primary ovarian clear cell tumors that should be distinguished from RCC metastasis: primary clear cell carcinoma, steroid cell tumors, and dysgerminoma [8, 11]. Because metastasis of RCC can mimic these entities, they concluded that immunohistochemical testing is necessary to differentiate primary ovarian lesions from RCC metastasis. A panel of immunohistochemical markers was then proposed by these authors to provide additional support for the diagnosis, including CD10, CK7, CA125, ER, PR, and CK8.8, as used in the present case. Use of the RCC antibody was also described, which may be of value when the differential diagnosis includes RCC, but the specificity of this marker has not yet been established as a single marker [8]. The use of a panel of antibodies, including 34ßE12 (a multi-CK antibody solution), CA125, ER, PR, and vimentin has also been suggested by Nolan et al. to help distinguish these two entities [12]. The majority of RCCs show restricted expression of low-molecular-weight cytokeratins and vimentin. High-molecular-weight cytokeratin and CK7 are rarely expressed in RCCs [13].

Our patient received interleukin-2 for her liver and lung metastases and showed a very good response. On the other hand, this treatment did not prevent the development of ovarian lesions. Valappil et al. also described the use of interleukin-2 to treat abdominal recurrence of renal pathology, leading to improved survival with stable disease, as in our case, but not preventing development of ovarian metastasis either [14].

Adjuvant therapy is poorly described in the literature. In all cases, patients underwent surgery to remove renal and ovarian masses. When the preoperative evaluation deemed it possible, total surgical excision, which should be aggressive to leave no residual disease, was able to cure $40-60 \%$ of patients [15]. In some cases, metastases could be surgically removed, with favorable prognostic factors including complete resectability and a long interval between the initial diagnosis and metastasis development [16].

The classic adjuvant treatment for advanced RCC was immunotherapy (interferon-alpha, interleukin-2), even if its effectiveness is still controversial [17]. A recent Cochrane review [18] found improved survival with interferon, but the greatest benefits were achieved in a very select group of patients, as reported by Rini et al. [17].

A progestational agent has also been used for very advanced symptomatic carcinoma, mostly for symptom palliation [19].

More recently, targeted therapies, such as anti-VEGF agents and mTOR-inhibiting drugs, have been extensively studied in the context of RCC treatment and have shown promising results in phase III trials [18].

Radiotherapy is not indicated for RCC.

\section{Conclusion}

Here we report the 16th case of ovarian metastasis from RCC, first appearing as a highly hemorrhagic tumor. 
Although some metastasis was initially detected among these 16 cases, leading to identification of the primary lesion, most of the metastatic spread $(69 \%, n=11 / 16)$ was discovered in the course of follow-up of the RCC treatment.

Immunostaining is an essential tool to differentiate the origin of clear cell lesions in the ovaries. The pathogenesis of ovarian metastasis is not well understood, and the laterality of these lesions and unique anatomy of the left ovarian veins are thought to be among the possible explanations. Advanced RCC must be well staged and, when possible, surgical treatment must be aggressive, with excision of all tumoral tissue. The metastasis itself may be removed in some cases, improving the prognosis if the lesion is totally excised.

Adjuvant treatments have classically entailed immunotherapy, but recent developments in targeted therapies are very encouraging, paving the way for new treatment approaches in the future.

Declaration of interest The authors report no conflicts of interest. The authors alone are responsible for the content and the writing of the paper.

\section{References}

1. Ulbright TM, Roth LM, Stehman FB (1984) Secondary ovarian neoplasia. Cancer 53:1164-1174

2. Cameron RI, Ashe P, O'Rourke DM, Foster H, McCluggage WG (2003) A panel of immunohistochemical stains assists in the distinction between ovarian and renal clear cell carcinoma. Int Gynecol Pathol 22(3):272-276

3. Stone WS (1916) Metastatic carcinoma of ovaries. Surg Gynecol Obstet 22:407-423

4. Hobbs JE, Vorder Brueggge CF, Wegner CR, Wintemute RW (1957) Bilateral ovarian metastasis from renal adenocarcinoma: report of a case and discussion of the pathogenesis. Obstet Gynecol 9:198-205

5. Gavallos G, Tawfik O, Herrell D, Langenstroer P (2003) Renalovarian axis: a case report and review. Urology 62:74918-74922

6. Karsh J (1951) Secondary malignant disease of the ovaries: a study of 72 autopsies. Am J Obstet Gynecol 61(1):154-160

7. Spencer JR, Eriksen B, Garnett JE (1993) Metastatic renal tumour presenting as an ovarian clear cell carcinoma. Urology 41(6):582-584

8. Hammock L, Ghorab Z, Gomez-Fernandez CR (2003) Metastatic renal cell carcinoma to the ovary: a case report and discussion of differential diagnoses. Arch Pathol Lab Med 127(3):123-126
9. Young RH, Hart WR (1992) Renal cell carcinoma metastatic to the ovary: a report of three cases emphasizing possible confusion with ovarian clear cell adenocarcinoma. Int J Gynecol Pathol 11 (20):96-104

10. Petru E, Pickel H, Heydarfadai M, Lahousen M, Haas J, Schaider $\mathrm{H}$ et al (1992) Nongenital cancers metastastic to the ovary. Gynecol Oncol 44:83-86

11. Insabato L, De Rosa G, Franco R, D'Onofrio V, Di Vizio D (2003) Ovarian metastasis from renal cell carcinoma: a report of three cases. Int J Surg Pathol 11(4):309-312

12. Nolan LP, Heatley MK (2001) The value of immunohistochemistry in distinguishing between clear cell renal carcinoma of the kidney and the ovary. Int J Gynecol Pathol 20:155-159

13. Skinnider B, Folpe A, Hennigar R, Lim S et al (2005) Distribution of cytokeratins and vimentin in adult renal neoplasms and normal renal tissue. Potential utility of a cytokeratin antibody panel in the differential diagnosis of renal tumors. Am J Surg Pathol 29:747754

14. Valappil SV, Toon PG, Anandaram PS (2004) Ovarian metastasis from primary renal cell carcinoma: report of a case and review of the literature. Gynecol Oncol 94:846-849

15. Margulis V, Sánchez-Ortiz RF, Tamboli P, Cohen DD, Swanson DA, Wood CG (2007) Renal cell carcinoma clinically involving adjacent organs: experience with aggressive surgical management. Cancer 109:2025-2030

16. Pfannschmidt J, Kugler C, Muley T, Hoffmann H, Dienemann H (2002) Prognostic factors for survival after pulmonary resection of metastatic renal cell carcinoma. Ann Thorac Surg 74:1653-1657

17. Rini BI, Campbell SC, Escudier B (2009) Renal cell carcinoma. Lancet 373:1119-1132

18. Coppin C, Porzsolt F, Awa A, Kumpf J, Coldman A, Wilt T (2005) Immunotherapy for advanced renal cell cancer. Cochrane Database Syst Rev 1:CD001425

19. Pizzocaro G, Di Fronzo G, Cappelletti V, Piva L, Salvioni R, Ronchi E et al (1983) Hormone treatment and sex steroid receptors in metastatic renal cell carcinoma: report of a multicentric prospective study. Tumori 69:215-220

20. Stefani P, Selli C, Nicita G, Turini D (1981) Angiographic aspects of renal clear cell carcinoma metastatic to the female genitalia. Cardiovasc Intervent Radiol 4:183-186

21. Buller RE, Braga CA, Tanagho EA, Miller T (1983) Renal cell carcinoma metastatic to the ovary: a case report. J Reprod Med 28:217-220

22. Adachi Y, Sasagawa I, Nakada T, Saitoh M, Mitobe K, Ohta T et al (1994) Bilateral ovarian metastasis from left renal cell carcinoma. Urol Int 52:169-171

23. Fields S, Libson E, Lavie O, Beller U (1993) Renal cell carcinoma metastatic to the ovary. Ultrasound and CT appearance. Clin Imaging 20:42-44

24. Vara A, Madrigal B, Veiga M, Diaz A, García J, Calvo J (1998) Bilateral ovarian metastasis from renal cell carcinoma. Acta Oncol 37(4):379-380

25. Stolnicu S, Borda A, Radulescu D, Puscasiu L, Berger N, Nogales FF (2007) Metastasis from papillary renal cell carcinoma masquerading as primary ovarian clear cell tumor. Pathol Res Practice 203:819-822 